\title{
DIAGNÓSTICO DE INTOLERANCIA A LA GLUCOSA, EN EL SERVICIO DE PATOLOGÍA CLÍNICA DEL HOSPITAL II SUÁREZ ANGAMOS - ESSALUD, 2010 LIMA - PERÚ
}

\author{
Luis Edgardo Figueroa Montes ${ }^{1}$ \\ 1. Servicio de Patología Clínica Hospital II Suárez Angamos de EsSalud Lima -Perú.
}

\section{RESUMEN}

Introducción: La diabetes mellitus tipo 2 se ha convertido en uno de los más graves problemas sanitarios de nuestro tiempo, y sus proporciones son ya epidémicas en la mayor parte del mundo. Existe por tanto un interés creciente en identificar individuos con alto riesgo de diabetes para planificar estrategias de prevención. Objetivo: Determinar el número de pacientes que acuden a realizarse un test de tolerancia oral a la glucosa y presentan intolerancia a la misma. Material y métodos: Se realizó un estudio descriptivo observacional en pacientes de ambos sexos, a quienes se les realizó el test de tolerancia oral a la glucosa, durante el año 2010, en la ciudad de Lima. Se utilizaron los criterios diagnósticos establecidos por la American Diabetes Association. El análisis de resultados se realizó utilizando el programa SPSS 18.0. Resultados: La edad promedio fue de 65 años. La mayoría, 1335 (71,3\%) fueron mujeres. De 1872 pacientes que ingresaron al estudio, el 88,5\% (1657) presentó glucemia alterada en ayunas y $215(11,5 \%)$, valores normales de glucemia. Un total de $486(26 \%)$ pacientes presentaron intolerancia a la glucosa. Conclusiones: El número de pacientes con intolerancia a la glucosa es elevado. La institución debería priorizar la atención de estos pacientes para que ingresen a un programa de prevención de diabetes y poder disminuir la incidencia y complicaciones de esta enfermedad que genera un gran gasto social. ( Rev. $m d$. panacea $2011 ; 1: 72-77$ ).

Palabras clave: diabetes mellitus, test de tolerancia a la glucosa, intolerancia a la glucosa. (Fuente: DeCS, BIREME)

\section{DIAGNOSIS OF GLUCOSE INTOLERANCE IN THE CLINICAL PATHOLOGY SERVICE AT HOSPITAL II SUÁREZ ANGAMOS - ESSALUD, 2010 LIMA - PERU}

\section{ABSTRACT}

Introduction: Diabetes mellitus type 2 has become one of the most serious sanitary problems of our time, and their proportions are already epidemic in most of the world. An increasing interest to identify individuals with high risk of diabetes exists therefore to plan prevention strategies. Objective: To determine the number of patients who perform a glucose tolerance test and present glucose intolerance. Materials and Methods: A descriptive observational study was realized, in patients, of both sexes, the test of oral tolerance to the glucose was realized to them, during year 2010 at Lima city. The diagnostic criteria established by the American Association Diabetes were used. The analysis of results was realized using program SPSS 18.0. Results: The average age was 65 years. The majority, $1335(71.3 \%)$ was women. Of 1872 patients who entered the study, the $88.5 \%$ (1657) presented glycemia altered in fasting and $215(11.5 \%)$ normal values of glycemia. A total of $486(26 \%)$ patient ones presented glucose intolerance. Conclusions: The number of patients with glucose intolerance is high. The institution would have to prioritize the attention of these patients so that they get in a diabetes prevention program and decrease the incidence and complications of this disease that generates a great social cost.

(Rev. $72-77$ ).

Key words: diabetes mellitus, glucose tolerance test, glucose intolerance. (Source: Mesh, NLM)

\section{INTRODUCCIÓN}

La diabetes mellitus tipo 2 (DM2) se ha convertido en uno de los más graves problemas sanitarios de nuestro tiempo, y sus proporciones son ya epidémicas en la mayor parte del mundo ${ }^{1}$. Existe por tanto un interés creciente en identificar individuos con alto riesgo de diabetes para planificar estrategias de prevención, ya que varios estudios han demostrado que con cambios en el estilo de vida y/o fármacos es posible prevenir 0 al menos retrasar la aparición de la enfermedad ${ }^{2,3,4,5}$.
La progresión desde la normoglucemia a la diabetes puede tardar varios años, lo que conlleva lógicamente estadios intermedios de disglucemia. Se engloban aquí dos categorías diagnósticas: la glucemia alterada en ayunas (GAA) y la intolerancia a la glucosa (ITG). El término de prediabetes fue acuñado por la Organización Mundial de la Salud (OMS) como un diagnóstico retrospectivo que describía el estado de una persona previamente al diagnóstico de diabetes 6 . Una definición radicalmente distinta ha sido recientemente introducida en Estados Unidos para describir colectivamente a los individuos con GAA y/o ITG, subrayando su alto riesgo de desarrollar diabetes en el futuro. Desde entonces, el uso de este término: prediabetes, se ha generalizado en la literatura científica $^{7,8}$. 
La ITG hace referencia a concentraciones de glucemia entre 140 y $199 \mathrm{mg} / \mathrm{dL}$ a las 2 horas después de una sobrecarga oral de glucosa, cifras que están claramente por encima de la normalidad pero por debajo del límite para definir diabetes. El concepto de GAA fue introducido en el informe de la American Diabetes Associatión (ADA) ${ }^{9}$ en 1997, con el objetivo de definir la zona entre el límite superior para la glucemia basal normal y el límite inferior de la glucemia basal asociada a la diabetes, como categoría análoga a la ITG para la glucemia basal, definiéndola como una concentración de glucemia en ayunas de entre 110 y $125 \mathrm{mg} / \mathrm{dL}$. En 2003, la ADA decidió reducir el límite inferior para definir la GAA a $100 \mathrm{mg} / \mathrm{dL}$, optimizando de este modo su sensibilidad y especificidad para predecir la diabetes ${ }^{10}$. En el contexto de la prevención de DM, cuando se presentan estos estados precoces de disglicemias (GAA e ITG), se ha sugerido que las estrategias de prevención, acorde a lo establecido por la ADA, deben ser focalizadas en aquellos casos que presentan las condiciones conjuntas de GAA e ITG. En el presente trabajo se aplicaron los criterios diagnósticos para prediabetes y diabetes según la $\mathrm{ADA}^{11}$.

Diferentes organizaciones resolvieron crear la Coalición Latinoamérica Saludable para la prevención y control de las enfermedades no transmisibles (ENT), como la diabetes mellitus, siendo la primera recomendación general: "Instrumentar políticas y acciones de promoción de la salud, prevención y control de las ENT en todos los sectores y niveles de gobierno asegurando la asignación de recursos necesarios para este fin" 12

En el Perú, la diabetes es una enfermedad que afecta a casi 2 millones de personas y es la décimo quinta causa de mortalidad, según informes de la Oficina de Estadística e Informática del Ministerio de Salud (2003); lo peor es que esta cifra va en aumento y se calcula que la mitad de los afectados ignora su condición. En EsSalud, sólo para el período 2009 , se han registrado un total de 26,090 casos de DM, significando una Tasa de Incidencia Acumulada de $250.8 \times 100,000$ asegurados ${ }^{13}$.

Conscientes de esta problemática, mi grupo de trabajo desea contribuir, mediante este estudio, con la solución de esta patología; diagnosticando oportunamente a los pacientes con intolerancia a la glucosa, debido a su mayor riesgo de desarrollar diabetes y enfermedades cardiovasculares, para que estos pacientes, en un futuro, puedan ser incluidos en un programa de prevención de diabetes ${ }^{14,15}$.

El objetivo de la presente investigación fue el de determinar el número de pacientes que acuden para realizarse un test de tolerancia oral a la glucosa (TTOG) y presentan ITG, en el servicio de Patología Clínica del Hospital II Suárez Angamos, Lima.

\section{MATERIAL Y MÉTODOS}

La población de estudio estuvo constituida por todos los pacientes que acudieron al servicio de Patología Clínica del Hospital II Suárez Angamos-EsSalud, 2010 Lima- Perú, para atenderse, de los cuales nuestra unidad de muestreo fueron los pacientes con solicitudes para realizarse el TTOG.

\section{Criterios de exclusión:}

1. Pacientes menores a 18 años.

2. Pacientes con solicitudes para descartar diabetes mellitus gestacional.

3. Pacientes que referían tener diagnóstico previo de diabetes mellitus.

4. Pacientes que presentaron valores de glucosa plasmática en ayunas $\geq 126 \mathrm{mg} / \mathrm{dl}$.

5. Pacientes que presentaron eventos adversos (náuseas, vómitos, mareos, otros) después de tomar la glucosa anhidra.

6. Pacientes que no acudieron a la toma de muestra de la glucosa a las 2 horas después de la sobrecarga oral de glucosa.

Se realizó un estudio transversal descriptivo, en un período comprendido entre los meses de enero y diciembre del 2010, en población asegurada de la ciudad de Lima. Se obtuvieron los siguientes datos: edad, sexo, centro asistencial de procedencia, especialidad médica que generó la solicitud, resultados de la medición de glucosa plasmática en ayunas (GPA) y la glucosa a las 2 horas. Todos los datos se registraron en una ficha de registro. Todo este procedimiento de dividió en tres etapas:

Etapa pre analítica: a los pacientes aptos se les tomó la primera muestra de la GPA, en tubos BD Vacutainer ${ }^{\circledR} \mathrm{K} 2$ EDTA $5.4 \mathrm{mg}$. Después se les administró 75 gramos de glucosa anhidra diluidos en $300 \mathrm{ml}$ de agua ${ }^{16}$. Luego se tomó la muestra de glucosa a las 2 horas.

Etapa analítica: las muestras de glucosa fueron procesadas en el analizador automatizado Hitachi 917, con el reactivo de glucosa GOD-PAP (Roche Diagnostics). Nuestro planeamiento de la calidad está enmarcado dentro de los lineamientos del Clinical and Laboratory Standards Institute, Clinical Laboratory Improvements Amendments y la American Society for Clinical Pathology ${ }^{17}$.

Etapa post analítica: los resultados fueron evaluados y liberados para su ingreso al sistema informático Telnet Win32 v2.0, 1999. Todos estos datos fueron recolectados en la ficha de registro e incluidos en una base de datos construida con el programa SPSS ${ }^{18}$, donde fueron procesados y analizados. Para el análisis univariado, se calcularon: las medias, desviaciones estándar, frecuencias y proporciones.

A todos los pacientes se les dio las indicaciones preanalíticas y se obtuvo el consentimiento verbal de los mismos, antes de la realización del TTOG previa explicación del procedimiento.

El autor declara no tener conflictos de intereses en relación con el contenido del presente estudio. 


\section{RESULTADOS}

En total fueron incluidos en el estudio 1872 pacientes. El promedio de edad de los mismos fue de $59.62 \pm 13,46$ años. Según la distribución por sexos, observamos que $1335(71,3 \%)$ eran mujeres y $537(28,7 \%)$ varones. Según la distribución por centro asistencial de origen tenemos que: $1507(80,5 \%)$ procedían del HSA, $145(7,8 \%)$ de la unidad básica de atención primaria Santa Cruz, $126(6,7 \%)$ del Policlínico Pablo Bermúdez y $94(5,0 \%)$ del Policlínico Próceres (Ver Tabla 1).

Tabla 1: Diagnóstico de intolerancia a la glucosa, según sexo y centro asistencial de origen. HSA II - EsSalud - Lima - Perú - 2010

\begin{tabular}{lrr}
\hline \multicolumn{1}{c}{ VARIABLES } & $\mathbf{n}$ & $\%$ \\
\hline Sexo & & \\
Femenino & 1335 & 71,3 \\
Masculino & 537 & 28,7 \\
Centro asistencial de origen & & \\
Hospital Suárez Angamos & 1507 & 80,5 \\
UBAP Santa Cruz & 145 & 7,8 \\
Policlínico Pablo Bermúdez & 126 & 6,7 \\
Policlínico Próceres & 94 & 5,0 \\
\hline Total & 1872 & 100,0 \\
\hline
\end{tabular}

Al evaluar los resultados de la glucosa plasmática en ayunas vemos que el $88,5 \%$ de pacientes presentaron glucemia alterada en ayunas y sólo el $11,5 \%$ valores normales de glucemia. Respecto a la glucemia 2 horas después de la sobrecarga oral de glucosa anhidra, 1295 $(69,2 \%)$ de los pacientes presentaron tolerancia normal a la glucosa, $486(26,0 \%)$ presentaron intolerancia a la glucosa y $91(4,8 \%)$ presentaron valores compatibles con diabetes (Tabla 2).

Tabla 2: Diagnóstico de intolerancia a la glucosa, según glucosa plasmática en ayunas y glucosa a las 2 horas.

HSA II - EsSalud - Lima - Perú - 2010

\begin{tabular}{lrr}
\hline \multicolumn{1}{c}{ VARIABLES } & $\mathbf{n}$ & $\%$ \\
\hline Glucosa plasmática en ayunas & 1657 & 88,5 \\
Glucemia alterada en ayunas (GAA) & 215 & 11,5 \\
Glucosa normal & & \\
Glucosa a las 2 horas & 1295 & 69,2 \\
Tolerancia normal a la glucosa & 486 & 26,0 \\
Intolerancia a la glucosa (ITG) & 91 & 4,8 \\
Diabetes mellitus & $\mathbf{1 8 7 2}$ & $\mathbf{1 0 0 , 0}$ \\
\hline Total & & \\
\hline
\end{tabular}

El total de pacientes que presentaron intolerancia a la glucosa y glucemia alterada en ayunas a la vez fueron 451 $(24,09 \%)$ del total de pacientes que acudieron a realizarse un TTOG. Además, 1657 pacientes presentaron glucemia alterada en ayunas y 451 intolerancia a la glucosa (Ver tabla 3).

Tabla 3: Diagnóstico de intolerancia a la glucosa, según glucemia alterada en ayunas, intolerancia a la glucosa o ambas.

HSA II - EsSalud - Lima - Perú - 2010

\begin{tabular}{lc}
\hline \multicolumn{1}{c}{ VARIABLES } & $\mathbf{n}$ \\
\hline Glucemia alterada en ayunas & 1657 \\
Intolerancia a la glucosa & 486 \\
GAA + ITG & 451 \\
\hline
\end{tabular}

\section{DISCUSIÓN}

Es conocido, que el TTOG, es el método más recomendado para evaluar a los pacientes que presentan una glucemia alterada en ayunas o aquellos pacientes con factores que lo condicionen, y así poder evaluar el riesgo de desarrollar diabetes mellitus tipo 2 (DM2), determinando su estado de glucemia ${ }^{11}$. Por ello, en el presente estudio se realizó el TTOG a todos los pacientes derivados por médicos de la consulta externa del HSA II, Policlínico Pablo Bermúdez, Policlínico los Próceres y la UBAP Santa Cruz de la Red Asistencial Rebagliati - EsSalud, a nuestro laboratorio.

En el HSA II, durante el 2010, los pacientes mayores de 45 años tuvieron un total de 291,274 atenciones en consulta externa, representando el $69,06 \%$ de todas las atenciones en ese año ${ }^{18}$. Un estudio previo demostró un elevado porcentaje de ITG en este grupo de pacientes ${ }^{19}$.

Múltiples estudios han investigado sobre la prevalencia de prediabetes (ITG + GAA) en población en general, pero pocos artículos publicados evalúan la presencia de intolerancia a la glucosa en pacientes que provienen de la consulta externa en centros asistenciales de salud.

El promedio de glucosa plasmática en ayunas fue de $109,6 \pm 8,15 \mathrm{mg} / \mathrm{dl}$ y de la glucosa a las 2 horas después de la sobrecarga oral de glucosa anhidra fue de $128,3 \pm 38,16$ $\mathrm{mg} / \mathrm{dl}$. La mayoría de los pacientes 1657 (88,5 \%) presentaron glucemia alterada en ayunas; sin embargo, solo $486(26 \%)$ pacientes presentaron intolerancia a la glucosa. Probablemente la mayoría de pacientes que acuden al laboratorio a realizarse este test, están aún en alguna etapa fisiopatológica inicial de la evolución hacia la DM $2{ }^{20}$. Como sabemos la GAA se caracteriza por un déficit en la fase de secreción precoz de insulina y por un aumento de la resistencia hepática a la acción de esta hormona, lo que se traduce en un incremento de la neoglucogenia hepática y la ITG se caracteriza por un aumento de la resistencia periférica a la acción de la insulina en el músculo, así como por un déficit de secreción 
de insulina, tanto en la fase precoz como en la tardía. Por lo tanto una intervención en este grupo de pacientes sería beneficiosa por su condición fisiopatológica ${ }^{21,22}$.

A diferencia de la mayoría de estudios donde se evalúa la prevalencia de intolerancia a la glucosa en poblaciones sanas donde el promedio de la misma esta entre 5 a $15 \%$, el presente estudio se realizó en pacientes con GAA, es por esta razón que el porcentaje de pacientes con ITG es de 26 $\%$. Diferentes estudios prospectivos disponibles muestran que tanto la ITG como la GAA son altamente predictivas de diabetes y que su efecto es acumulativo, observándose un riesgo máximo en los individuos con GAA e ITG combinadas. La inclusión de ambas mediciones permite una mejor estratificación del riesgo de diabetes ${ }^{23}$.

Observamos que el 92,79\% (451) de los pacientes con ITG presentaban GAA, por lo tanto conociendo que el riesgo de desarrollar DM 2 en pacientes con ITG o GAA es similar (y se estima que es 4-5 veces mayor que en los sujetos con tolerancia normal a la glucosa), con una tasa de desarrollo anual de diabetes del $5-10 \%$. En aquellas personas que presentan GAA+ITG, el riesgo es aproximadamente el doble que con cualquiera de ellas aisladamente 23,24

Nosotros, en su estudio previo, evaluamos el diagnóstico de ITG en pacientes mayores de 50 años en un hospital, en población asegurada de Lima-Perú; encontramos un 28,3\% de ITG. En este estudio se recomendó la implementación de un programa de prevención de diabetes en la Red Asistencial Rebagliati de Essalud ${ }^{19}$.

Zubiate y col. al evaluar obesidad, intolerancia a la glucosa y diabetes mellitus en la población adulta general de Lima, encuentra que las prevalencias de ITG y de DM son parecidas entre sí, con discreto predominio en el sexo masculino. Además observa los vínculos entre ITG y DM con la edad, antecedentes familiares de diabetes e HTA son evidentes ${ }^{25}$.

García y col. en su estudio donde evalúa la prevalencia de diabetes mellitus y factores de riesgo relacionados en una población urbana de Lima-Perú, encuentra una prevalencia de intolerancia a los carbohidratos de 2,82\% (IC95\%: 0,6\%$5,04 \%)$ y de glucemia alterada en ayunas de $17,84 \%$ (IC95\%:12,70\%-22,98\%) ${ }^{26}$.

Otros estudios han demostrado que tanto la GAA como la ITG implican un riesgo cardiovascular (RCV) aumentado. Sin embargo, la ITG conlleva un riesgo más elevado y es un mejor factor pronóstico del riesgo cardiovascular que la GAA $27,28,29$.

Por todas estas razones la atención de esta población es prioritaria, para que la institución realice algún tipo de intervención orientada a modificar sus estilos de vida y así disminuir la probabilidad de que desarrollen enfermedades cardiovasculares o DM 2. Esta evidencia meta analizada en pacientes identificados con ITG, demuestra eficacia y rentabilidad en la aplicación de políticas de prevención para el retardo de la aparición de DM 2. Deberían considerarse diversas estrategias de identificación y de investigación en esta población ${ }^{30}$.

Del mismo modo, asumiendo un escenario hipotético, estos pacientes con ITG, retornarían a su atención en la consulta externa, serian evaluados y el médico habría indicado al paciente las intervenciones necesarias para modificar su prediabetes. Sin embargo, un estudio demostró que la mayoría de estos pacientes no eran diagnosticados y tratados adecuadamente ${ }^{31}$.

La DM 2 es un problema de salud cada vez mayor, con el predominio dramático de esta enfermedad en sociedades occidentalizadas. Los individuos con DM2 tienen una esperanza de vida que se acorta aproximadamente en 15 años, principalmente por las complicaciones macrovasculares ${ }^{32}$.

El aumento epidémico de la DM y sus consecuencias a largo plazo justifican los esfuerzos para prevenir su aparición. Es importante identificar a los individuos con alto riesgo de desarrollar diabetes para prevenir el empeoramiento de la hiperglucemia. Por lo tanto, es necesario hacer un doble esfuerzo; por un lado, identificar a los pacientes de mayor riesgo para desarrollar diabetes $y$, por otro, intervenir con un programa de prevención de DM.

A partir de nuestros resultados y en concordancia con la evidencia actual, esperamos que las recomendaciones puedan aplicarse en nuestra institución.

\section{Correspondencia:}

Figueroa Montes, Luis Edgardo.

Correo electrónico: patologoclinico@gmail.com

Teléfono: 986659670

Dirección: Jiron Irribarren N No 769 Dpto 404

$$
\text { Surquillo - Lima }
$$

\section{Agradecimientos:}

Al Dr. Miranda-Soberón Ubaldo, Pediatra investigador del Hospital Docente de Ica, docente de UNSLG - Ica, por su colaboración.

\section{REFERENCIAS BIBLIOGRÁFICAS}

1. International Diabetes Federation. Diabetes Atlas, 3rd ed. IDF, 2006.

2. Tuomilehto J, Lindstrom J, Eriksson JG, Valle TT, Hamalainen $H$, llanne Parikka $P$, et al. Prevention of type 2 diabetes mellitus by changes in lifestyle among subjects with impaired glucose tolerance. $\mathrm{N}$ Engl J Med. 2001; 344:1343-50.

3. Diabetes Prevention Program Research Group. Reduction in the incidence of type 2 diabetes with lifestyle intervention or metformin. $\mathrm{N}$ Engl J Med. 2002; 346:393-403. 
4. Chiasson J, Josse R, Gomis G, Hanefi eld R, Karasik M, Laakso A. Acarbose for prevention of type 2 diabetes mellitus: the STOP-NIDDM randomised trial. Lancet. 2002; 359:2072-7.

5. The DREAM trial investigators. Effect of rosiglitazone on the frequency of diabetes in patients with impaired glucose tolerance or impaired fasting glucose: a randomised controlled trial. Lancet. 2006; 368:1096-105.

6. Jackson WP. That expression, "prediabetes". Diabetes. 1962; 11:334.

7. US Department of Health and Human Services, American Diabetes Association. HHS, ADA warn Americans of "pre-diabetes". Press release. 27 Mar 2002. Washington, DC: DHHS, 2002.

8. Unwin $\mathrm{N}$, Shaw J, Zimmet $\mathrm{P}$, Alberti KGMM. Impaired glucose tolerance and impaired fasting glycemia: the current status on definition and intervention. Diabet Med. 2002; 19:708-23.

9. World Health Organization. Definition, diagnosis and classification of diabetes mellitus and its complications: report of a WHO consultation. Part 1: Diagnosis and classification of diabetes mellitus. Geneva: WHO, 1999.

10. American Diabetes Association. The Expert Committee on the Diagnosis and Classification of Diabetes Mellitus. Follow-up report on the diagnosis of diabetes mellitus. Diabetes Care. 2003; 26:3160-7.

11. Diagnosis and Classification of Diabetes Mellitus. American Diabetes Association. Diabetes Care, vol 34, supplement, January 2011. Pgs. 562-569.

12. Asociación latinoamericana de diabetes. Declaración latinoamericana frente a la emergencia sanitaria de las Enfermedades No Trasmisibles (ENT). Marzo 2011 Buenos Aires Argentina. Available from: http://www.alad latinoamerica.org/declaracion\%20f inal $\% 204 \% 20 \mathrm{de} \% 20$ marzo $\% 202011$.pdf.

13. Resolución de Gerencia General de Prestaciones de Salud No 066 - GCPS - EsSalud - 2010. Guía de práctica clínica de diabetes mellitus tipo 2 para el primer nivel de atención. Agosto 2010-EsSalud.

14. Directiva $N^{\circ} 02$ GCPS - EsSalud - 2010. Programa de atención integral rápida al paciente con enfermedad crónica estable en EsSalud (PAIRPEC) Red Asistencial Rebagliati.

15. Resolución de Gerencia General № 262 - GG ESSALUD - 2010. Plan Nacional de Prevención EsSalud 2010. Marzo 2010
16. Guías $A L A D$ de diagnóstico, control y tratamiento de la Diabetes Mellitus Tipo 2. Versión 2000.

17. Basic Method Validation, 3rd Edition. James $O$. Westgard, PhD. Published by Westgard QC, Inc. 2008.

18. Perfil de la demanda en consulta externa 2010. Área de estadística del Hospital Suárez Angamos II. EsSalud. 2010

19. Figueroa y col. Diagnóstico de intolerancia a la glucosa en pacientes mayores de 50 años, en el servicio de laboratorio del Hospital Suárez Angamos II - Essalud Lima 2010. Resolución de presidencia ejecutiva № 389 PE-ESSALUD - 2011.

20. Sociedad argentina de diabetes. Consenso sobre criterio diagnostico de la glucemia alterada en ayunas. Revista de la Sociedad Argentina de Diabetes, 41(3): 2007.

21. David M. Nathan, Mayer B. Davidson, Ralph A. De Fronzo, et al. Impaired Fasting Glucose and Impaired Glucose Tolerance: Implications for care Diabetes Care. March 2007. 30:753-759.

22. Meyer C, Pimenta W, Woerle HJ, Van Haeften T, Szoke E, Mitrakou A, et al. Different mechanisms for impaired fasting glucose and impaired postprandial glucose tolerance in humans. Diabetes Care. 2006; 29:1909-14.

23. Qiao $Q$, Lindström J, Valle TT, Tuomilehto J. Progression to clinically diagnosed and treated diabetes from impaired glucose tolerance and impaired fasting glycaemia. Diabet Med. 2003; 20:1027-33.

24. Rasmussen SS, Glümer C, Sandbaek A, Lauritzen $\mathrm{T}$, Borch-Johnsen K. Determinants of progression from impaired fasting glucose and impaired glucose tolerance to diabetes in a high-risk screened population: 3 year follow-up in the ADDITION study, Denmark. Diabetologia. 2008; 51:249-57.

25. Zubiate Mario; Valdivia Freddy; Díaz Elssie; Millones Bernardo; Aliaga Elsi, et al. Obesidad, intolerancia a la glucosa y diabetes mellitus en la población adulta general de Lima. Rev. méd. Inst. Peru. Segur. Soc; 4(2):7-17, abr.-jun. 1995.

26. García, José Solís, Jorge Calderón, Edith Luque, Luis Neyra, et al. Prevalencia de diabetes mellitus y factores de riesgo relacionados en una población urbana. Rev Soc Peru Med Interna 2007; 20(3).

27. Coutinho M, Gerstein HC, Wang Y, Yusuf S. The relationship between glucose and incident cardiovascular events. A metaregression analysis 
28. of published data from 20 studies of 95,783 individuals followed for 12.4 years. Diabetes Care. 1999; 22:233-40.

29. Levitzky YS, Pencina MJ, D’Agostino RB, Meigs JB, Murabito JM, Vasan RS, et al. Impact of impaired fasting glucose on cardiovascular disease: the Framingham Heart Study. J Am Coll Cardiol. 2008; 51:264-70.

30. Earl S. Ford, Guixiang Zhao, Chaoyang Li. PreDiabetes and the Risk for Cardiovascular Disease A Systematic Review of the Evidence JACC. 55(13):1310-7. March 30, 2010

31. Clare L Gillies, Keith R Abrams, Paul C Lambert, Nicola J Cooper, Alex J Sutton, Ron T Hsu, et al. Pharmacological and lifestyle interventions to prevent or delay type 2 diabetes in people with impaired glucose tolerance: systematic review and meta-analysis. February 2007 Volume 334, Issue
7588 BMJ, doi: 10.1136 / bmj.39063.689375.55. 2007.

32. Karve A, Hayward RA. Prevalence, Diagnosis, and Treatment of Impaired Fasting Glucose and Impaired Glucose Tolerance in Nondiabetic U.S. Adults. Diabetes Care; 33(11):2355-9. Nov 2010.

33. Davies MJ, Tringham JR, Troughton J, Khunti KK Prevention of type 2 diabetes mellitus. A review of the evidence and its application in a UK setting Diabet Med 2004; 21:403-14.

Recibido: 23/10/2012

Aceptado para publicación: 24/02/2012 